\title{
Wave functions for arbitrary operator ordering in the de Sitter minisuperspace approximation ${ }^{\dagger}$
}

\author{
David L. Wiltshire* \\ Department of Physics and Mathematical Physics, University of Adelaide, Adelaide S.A. 5005, \\ Australia.
}

ADP-99-22/M81, gr-qc/9905090

\begin{abstract}
We derive exact series solutions for the Wheeler-DeWitt equation corresponding to a spatially closed Friedmann-Robertson-Walker universe with cosmological constant for arbitrary operator ordering of the scale factor of the universe. The resulting wave functions are those relevant to the approximation which has been widely used in two-dimensional minisuperspace models with an inflationary scalar field for the purpose of predicting the period of inflation which results from competing boundary condition proposals for the wave function of the universe. The problem that Vilenkin's tunneling wave function is not normalizable for general operator orderings, is shown to persist for other values of the spatial curvature, and when additional matter degrees of freedom such as radiation are included.
\end{abstract}

$\dagger$ Based on a talk presented at the 2nd Australasian Conference on General Relativity and Gravitation, Sydney, July 1998.

To appear with the Proceedings in General Relativity and Gravitation

* Electronic address: dlw@physics.adelaide.edu.au

\section{INTRODUCTION}

Over the past year new developments concerning the possibility of open inflation [1.2] in quantum cosmology have led to renewed interest in the debate [2 4 i] about the relative merits of competing proposals for boundary conditions on the wave function of the Universe. This debate has centred on the differing predictions of the "no boundary" proposal of Hartle and Hawking [5], and the "tunneling" proposals of Vilenkin [6.7] and of Linde [8], which are themselves distinct.

Much of the debate between proponents of the competing proposals arises from differing predictions obtained using probability amplitudes which are assumed to apply to the 
respective wave functions. In particular, the nucleation probability for instanton-dominated transitions which describe a universe which "tunnels from nothing" is assumed to be

$$
\mathcal{P} \propto|\Psi|^{2} \propto \begin{cases}\mathrm{e}^{-2 I_{\mathrm{cl}}} & \Psi_{\mathrm{NB}} \\ \mathrm{e}^{+2 I_{\mathrm{cl}}} & \Psi_{\mathrm{TL}}, \Psi_{\mathrm{TV}}\end{cases}
$$

where the subscripts (NB), (TL) and (TV) refer to the no-boundary wave function and the tunneling wave functions of Linde and Vilenkin respectively. For the solutions in question, which correspond to a model in which gravity is coupled to a scalar field, $\phi$, with potential, $V(\phi)$ in dimensionless units (see (3) below for our conventions), the Euclidean action of the instanton is

$$
I_{\mathrm{cl}}=\frac{-1}{3 V\left(\phi_{0}\right)},
$$

$\phi_{0}$ being the value of the scalar field at nucleation.

It has been pointed out by supporters of Vilenkin's proposal [4, 10, that arguments which use (11) as their starting point are sometimes ill-founded, since the identifications assumed in (1) are not supported by more detailed minisuperspace calculations in models such as that of black hole nucleation in the very early universe [9,10]. In particular, it appears that $\Psi_{\mathrm{TV}}$ cannot be identified with the probability amplitude (1) in this case, thereby side-stepping the criticism of [10]. We have pointed out recently [11], however, that similar criticisms apply to the model involving the nucleation of a closed Friedmann-Robertson-Walker universe with an inflationary scalar field - the "work horse model" of numerous discussions in quantum cosmology.

The conclusion drawn in [11] was that the identification of $\Psi_{\mathrm{TV}}$, (or indeed of $\Psi_{\mathrm{TL}}$ ), with the probability amplitude (11) depends on Planck scale physics through the choice of operator ordering in the minisuperspace Wheeler-DeWitt equation. This would not be a serious problem if there was a natural choice of operator ordering for which the identification (1) could still be made in the case of $\Psi_{\mathrm{TV}}$. However, the choice of ordering which to our knowledge is the only one to have been claimed as natural in this minisuperspace model 12 14, is one for which $\Psi_{\mathrm{TV}}$ cannot be normalized, the choice of normalization being central to the identification (11). The grounds for "naturalness" of the relevant factor ordering include 1-loop unitarity [14]. In fact, the same operator ordering was assumed in calculations which claim to lead to the suppression of values of the scalar field with a potential, $V(\phi)$, above the Planck scale in the relevant probability amplitude [15] - or even to lead to an enhancement of sub-Planckian values of $V(\phi)$ in a conformally coupled model [16] - arguments which have usually been assumed [7, 15, [16] to favour Vilenkin's tunneling wave function, $\Psi_{\mathrm{TV}}$. Our observation [11], which concerns Planck scale corrections in the scale factor, $a$, rather than in the scalar field $\phi$ (as assumed in [14 [16]), casts considerable doubt on such claims. Thus the "prediction of inflation" cannot be claimed as a success of Vilenkin's wave function, as is often widely assumed.

The conclusions of ref. [11] were based on an analysis of the second order ordinary differential equation obtained in the "de Sitter minisuperspace approximation" for arbitrary 
ordering of the scale factor in the Wheeler-DeWitt equation. Our analysis was based on the qualitative behaviour of this equation in various relevant limits. In fact, exact series solutions may be developed for the models of interest. It is our intention to present these solutions in this paper, and to relate the solutions to the results of [11.

\section{MINISUPERSPACE MODEL}

The specific model considered here is the 2-dimensional minisuperspace corresponding to the classical action for gravity coupled to a scalar field,

$$
S=\frac{1}{4 \kappa^{2}}\left[\int_{\mathcal{M}} \mathrm{d}^{4} x \sqrt{-g} \mathcal{R}+2 \int_{\partial \mathcal{M}} \mathrm{d}^{3} x \sqrt{h} \mathcal{K}\right]+\frac{3}{\kappa^{2}} \int_{\mathcal{M}} \mathrm{d}^{4} x \sqrt{-g}\left(-\frac{1}{2} g^{\mu \nu} \partial_{\mu} \phi \partial_{\nu} \phi-\frac{V(\phi)}{2 \sigma^{2}}\right)
$$

where $\kappa^{2}=4 \pi G=4 \pi m_{\text {Planck }}^{-2}, \mathcal{K}$ is the trace of the extrinsic curvature, and the metric is assumed to take the closed Friedmann-Robertson-Walker form

$$
\mathrm{d} s^{2}=\sigma^{2}\left\{-\mathcal{N}^{2} \mathrm{~d} t^{2}+a^{2}(t) \mathrm{d} \Omega_{3}^{2}\right\}
$$

where $\mathrm{d} \Omega_{3}{ }^{2}$ is a round metric on the 3-sphere, and $\sigma^{2}=\kappa^{2} /\left(6 \pi^{2}\right)$.

The Hamiltonian constraint obtained from the $(3+1)$-decomposition of the field equations may be quantized to yield the Wheeler-DeWitt equation

$$
\left[\frac{1}{a^{p}} \frac{\partial}{\partial a} a^{p} \frac{\partial}{\partial a}-\frac{1}{a^{2}} \frac{\partial^{2}}{\partial \phi^{2}}-a^{2} \mathcal{U}(a, \phi)\right] \Psi=0
$$

where

$$
\mathcal{U}(a, \phi) \equiv 1-a^{2} V(\phi)
$$

and we have allowed for possible operator-ordering ambiguities through the integer power, $p$, in the first term. The approximation that has been adopted in discussions such as that of the prediction of inflation from quantum cosmology [6,7] is to confine the discussion to regions in which the the potential $V(\phi)$ can be approximated by a cosmological constant, so that the $\phi$ dependence in (5) can be effectively ignored and a standard 1-dimensional WKB analysis applied.

The Wheeler-DeWitt equation for the "de Sitter minisusperspace approximation" has been solved exactly in terms of Airy functions by Vilenkin [7] for the special case of operator ordering $p=-1$. We wish to observe here that similar series solutions can be readily obtained for the case of arbitrary factor ordering. In particular, with the redefinition

$$
\Psi \equiv z^{-(p-1) / 4} y(z)
$$

where $z \equiv \frac{1}{2} a^{2}$, we find that the 1 -dimensional Wheeler-DeWitt resulting from (5) when the $\phi$-dependence is neglected is given by 


$$
z^{2} \frac{\mathrm{d}^{2} y}{\mathrm{~d} z^{2}}+z \frac{\mathrm{d} y}{\mathrm{~d} z}+\left(2 V z^{3}-z^{2}-\nu^{2}\right) y=0
$$

where $\nu=\left|\frac{1}{4}(p-1)\right|$. This equation is classified as a type $\{25\}$ Bôcher equation in the terminology of Moon and Spencer [17, although its solution has not been explicitly tabulated. Near the regular singular point at $z=0$, the solution $y(z)$ approaches that of the modified Bessel equation, since for finite $V$ the term involving $V$ in (8) is sub-dominant.

\section{CONVERGENT SERIES SOLUTIONS}

It is straightforward to apply the Frobenius method to (8) to obtain series solutions, although the calculations are rather lengthy. In particular, after a considerable amount of

algebra we find that the two linearly independent solutions may be constructed in terms of the power series $y_{+}(z)$ and $y_{-}(z)$ given by

$$
y_{ \pm}(z)=z^{ \pm \nu}\left\{1+\sum_{\ell=1}^{\infty}\left[\mathcal{A}_{2 \ell}^{ \pm} z^{2 \ell}-\mathcal{A}_{2 \ell+1}^{ \pm} z^{2 \ell+1}\right]\right\}
$$

where the coefficients of the even and odd powers of $z$ are respectively

$$
\begin{gathered}
\mathcal{A}_{2 \ell}^{ \pm}=\sum_{i=0}^{[\ell / 3]}(2 V)^{2 i} \boldsymbol{S}_{ \pm, \ell}^{2 i} \\
\mathcal{A}_{2 \ell+1}^{ \pm}=\sum_{i=0}^{[(\ell-1) / 3]}(2 V)^{2 i+1} \boldsymbol{\mathcal { S }}_{ \pm, \ell}^{2 i+1}
\end{gathered}
$$

[ ] denotes the integer part, and the sums $\boldsymbol{S}_{ \pm, \ell}^{j}$ are defined iteratively by

$$
\begin{aligned}
\boldsymbol{S}_{ \pm, \ell}^{0} & =\mathcal{C}_{ \pm, 2 \ell}^{0}, \quad \quad \ell \geq 0, & \\
\mathcal{S}_{ \pm, \ell}^{2 i+1} & =\sum_{j=3 i}^{\ell-1} \mathcal{C}_{ \pm, 2 \ell+1}^{2 j+1} \boldsymbol{S}_{ \pm, j}^{2 i}, & \ell \geq 1, \quad 0 \leq i \leq[(\ell-1) / 3] \\
\mathcal{S}_{ \pm, \ell}^{2 i} & =\sum_{j=3 i-1}^{\ell-1} \boldsymbol{\mathcal { C }}_{ \pm, 2 \ell}^{2 j} \boldsymbol{\mathcal { S }}_{ \pm, j-1}^{2 i-1} & \ell \geq 3, \quad 0 \leq i \leq[\ell / 3],
\end{aligned}
$$

in terms of the quantities $\mathbf{C}_{ \pm, \ell}^{j}$, defined by

$$
\mathbf{C}_{+, k}^{j}=\frac{j ! !(j+2 \nu) ! !}{k ! !(k+2 \nu) ! !} \equiv \frac{\Gamma\left(\frac{j}{2}+1\right) \Gamma\left(\frac{j}{2}+\nu+1\right)}{2^{k-j} \Gamma\left(\frac{k}{2}+1\right) \Gamma\left(\frac{k}{2}+\nu+1\right)}, \quad \nu \geq 0
$$

and 


$$
\mathbf{C}_{-, k}^{j}=\frac{j ! !(j-2 \nu) ! !}{k ! !(k-2 \nu) ! !} \equiv \frac{\Gamma\left(\frac{j}{2}+1\right) \Gamma\left(\frac{j}{2}-\nu+1\right)}{2^{k-j} \Gamma\left(\frac{k}{2}+1\right) \Gamma\left(\frac{k}{2}-\nu+1\right)}, \quad \nu>0, \quad \nu \neq 1, \frac{3}{2}, 2, \frac{5}{2}, \ldots
$$

$j$ and $k$ being two positive integers which differ by an even number. For the special cases $\nu=1, \frac{3}{2}, 2, \frac{5}{2}, \ldots$, the quantities (17) must be replaced by

$$
\mathfrak{C}_{-, k}^{j}= \begin{cases}\frac{(-1)^{(k-j) / 2} \Gamma\left(\frac{j}{2}+1\right) \Gamma\left(\nu-\frac{k}{2}\right)}{2^{k-j} \Gamma\left(\frac{k}{2}+1\right) \Gamma\left(\nu-\frac{j}{2}\right)}, & \text { if } k<2 \nu ; \text { or } k>2 \nu \text { and } 2 \nu-k \text { is odd, } \\ 0, & \text { if } k=2 \nu ; \text { or } k>2 \nu, j<2 \nu \text { and } 2 \nu-k \text { is even, } \\ \frac{\Gamma\left(\frac{j}{2}+1\right) \Gamma\left(\frac{j}{2}-\nu+1\right)}{2^{k-j} \Gamma\left(\frac{k}{2}+1\right) \Gamma\left(\frac{k}{2}-\nu+1\right)}, & \text { if } k>2 \nu, j \geq 2 \nu \text { and } 2 \nu-k \text { is even, }\end{cases}
$$

The linearly independent solutions to (8) are then

$$
Y_{1}(z)=y_{+}(z)
$$

and

$$
Y_{2}(z)= \begin{cases}y_{-}(z), & \nu \neq 0,1, \frac{3}{2}, 2, \frac{5}{2}, \ldots \\ y_{*}(z), & \nu=0 \\ y_{-}(z)+\mathcal{B}_{-} y_{*}(z), & \nu=1, \frac{3}{2}, 2, \frac{5}{2}, \ldots\end{cases}
$$

where

$$
\mathcal{B}_{-}=\frac{1}{2 \nu}\left(\mathcal{A}_{2 \nu-2}^{-}-2 V \mathcal{A}_{2 \nu-3}^{-}\right), \quad \nu \geq 1
$$

(with $\mathcal{A}_{0}^{-}=1, \mathcal{A}_{ \pm 1}^{-}=0$ ), and the series $y_{*}(z)$ is defined by

$$
y_{*}(z)=y_{+}(z) \ln (z)-2 \sum_{n=2}^{\infty} \sum_{i=1}^{h_{n}} \frac{(-2 V)^{n-2 \mathcal{D}_{n, i}}}{\prod_{j=1}^{\mathcal{D}_{n, i}} \alpha_{j}^{i}\left(\alpha_{j}^{i}+2 \nu\right)}\left(\sum_{k=1}^{\mathcal{D}_{n, i}} \frac{\left(\alpha_{k}^{i}+\nu\right)}{\alpha_{k}^{i}\left(\alpha_{k}^{i}+2 \nu\right)}\right) z^{n+\nu} .
$$

The quantities in the summation in the coefficient of $z^{n+\nu}$ in (22) are defined in terms of the sequences of all partial sums obtained from partitioning the integer $n$ by 2 or 3 . In particular, let

$$
\mathcal{P}_{n, i}=\left\{\alpha_{j}^{i} \in \mathbb{Z} \mid \alpha_{1}^{i}<\alpha_{2}^{i}<\ldots<\alpha_{\mathcal{D}_{n, i}^{i}}^{i}=n, \alpha_{1}^{i} \in\{2,3\}, \quad\left(\alpha_{j+1}^{i}-\alpha_{j}^{i}\right) \in\{2,3\}\right\}
$$

where $\mathcal{D}_{n, i}=\operatorname{dim} \mathcal{P}_{n, i}$. The index $i=1,2, \ldots, h_{n}$ labels the different possible distinct ordered sequences of such integers, the number of which is given by 


$$
h_{n}=\sum_{\substack{r=0, s=0 \\ 3 r+2 s=n}}^{[n / 3],[n / 2]} \frac{(r+s) !}{r ! s !}
$$

i.e., the $n$th element of the Padovan sequence [18,19], $\{1,0,1,1,1,2,2,3, \ldots\}$, defined recursively by $h_{0}=h_{2}=1, h_{1}=0, h_{n}=h_{n-3}+h_{n-2}$ for $n \geq 3$. The sequences in $\mathcal{P}_{n}$ may be constructed iteratively by simply appending the integer $\{n\}$ to each sequence in $\mathcal{P}_{n-3}$ and in $\mathcal{P}_{n-2}$, as shown in Table 1.

For $p=1$, (i.e., $\nu=0$ ), for example, we find solutions given by (19) and (20) with

$$
\begin{aligned}
y_{+}(z)=1 & +\frac{1}{4} z^{2}-\frac{1}{9} 2 V z^{3}+\frac{1}{64} z^{4}-\frac{13}{900} 2 V z^{5}+\left[\frac{1}{2304}+\frac{1}{324}(2 V)^{2}\right] z^{6}-\frac{433}{705600} 2 V z^{7} \\
& +\left[\frac{1}{147456}+\frac{71}{259200}(2 V)^{2}\right] z^{8}-\left[\frac{2957}{228614400}+\frac{1}{26244}(2 V)^{2}\right] 2 V z^{9} \\
& +\left[\frac{1}{14745600}+\frac{11273}{1270080000}\right](2 V)^{2} z^{10}+\ldots \\
y_{*}(z)=y_{+} & (z) \ln (z)-\frac{1}{4} z^{2}+\frac{2}{27} 2 V z^{3}-\frac{3}{128} z^{4}+\frac{253}{13500} 2 V z^{5}-\left[\frac{11}{13824}+\frac{1}{324}(2 V)^{2}\right] z^{6} \\
& +\frac{153527}{148176000} 2 V z^{7}-\left[\frac{25}{1769472}+\frac{2123}{5184000}(2 V)^{2}\right] z^{8}+\left[\frac{3671179}{144027072000}+\frac{11}{236196}(2 V)^{2}\right] 2 V z^{9} \\
& -\left[\frac{137}{884736000}+\frac{2886157}{177811200000}(2 V)^{2}\right] z^{10}+\ldots
\end{aligned}
$$

TABLE I. Integer sequences contributing to $y_{*}$

\begin{tabular}{lll}
\hline \hline$n$ & $h_{n}$ & $\mathcal{P}_{n, i}, i=1, \ldots, h_{n}$ \\
\hline 2 & 1 & $\{2\}$ \\
3 & 1 & $\{3\}$ \\
4 & 1 & $\{2,4\}$ \\
5 & 2 & $\{2,5\},\{3,5\}$ \\
6 & 2 & $\{3,6\},\{2,4,6\}$ \\
7 & 3 & $\{2,4,7\},\{2,5,7\},\{3,5,7\}$ \\
8 & 4 & $\{2,5,8\},\{3,5,8\},\{3,6,8\},\{2,4,6,8\}$ \\
9 & 5 & $\{3,6,9\},\{2,4,6,9\},\{2,4,7,9\},\{2,5,7,9\},\{3,5,7,9\}$ \\
10 & 7 & $\{2,4,7,10\},\{2,5,7,10\},\{3,5,7,10\},\{2,5,8,10\},\{3,5,8,10\},\{3,6,8,10\},\{2,4,6,8,10\}$ \\
$\vdots$ & $\vdots$ & $\vdots$ \\
\hline \hline
\end{tabular}




\section{ASYMPTOTIC SERIES AND THE WKB LIMIT}

Although the series solutions of Sec. III converge for all positive values of $a$, the rate of convergence is extremely slow and for many purposes a knowledge of the asymptotic series is desirable. For example, boundary conditions are often formulated in terms of the WKB solutions, which are effectively the leading order terms in such limits. Since the nature of the WKB solutions varies according to the sign of $\mathcal{U}(a, \phi)(6)$, instead of using the variable $z$ of Sec. III, it is convenient to introduce a new variable

$$
x \equiv(2 V)^{-2 / 3}(1-2 V z),
$$

and to make the transformation $y \equiv z^{-1 / 2} u$. We then see that (8) may be written equivalently as

$$
\frac{\mathrm{d}^{2} u}{\mathrm{~d} x^{2}}-\left[x+\frac{(2 V)^{4 / 3} \mu}{\left[(2 V)^{2 / 3} x-1\right]^{2}}\right] u=0
$$

where

$$
\mu \equiv \nu^{2}-\frac{1}{4}=\frac{1}{16}(p+1)(p-3)
$$

The essential point to note is of course that to leading order the asymptotic series derived from (28) as $x \rightarrow \pm \infty$ are no different to those of the special case $\mu=0$, or $p=-1$, for which $u(x)$ is an exact Airy function [7].

The first limit of interest is $x \rightarrow \infty$, which, when physically relevant, corresponds to large finite values of $a$ such that $a^{2} V \ll 1$ nonetheless. This requires us to take $V \ll 1$, i.e., to restrict the scalar field, $\phi$, to values with a potential much below the Planck scale, which is of course physically justified.

Since $\Psi \equiv\left(\frac{1}{2} a^{2}\right)^{-(p+1) / 4} u(x)$, we find that the appropriate asymptotic series for $\Psi$ are linear combinations of the two modes

$$
\begin{aligned}
\Psi_{ \pm} \propto \frac{\exp \left[\frac{ \pm \mathcal{U}^{3 / 2}}{3 V}\right]}{a^{(p+1) / 2} \mathcal{U}^{1 / 4}}\{1 \pm & \left(\frac{5}{48}-\frac{1}{3} \mu\right) \frac{2 V}{\mathcal{U}^{3 / 2}} \mp \frac{2}{5} \mu \frac{2 V}{\mathcal{U}^{5 / 2}} \\
& \left.+\left(\frac{77}{96}-\frac{1}{6} \mu\right)\left(\frac{5}{48}-\frac{1}{3} \mu\right) \frac{(2 V)^{2}}{\mathcal{U}^{3}} \mp \frac{3}{7} \mu \frac{2 V}{\mathcal{U}^{7 / 2}}+\ldots\right\}
\end{aligned}
$$

The leading order term is recognized as the WKB mode in the tunneling region. In practice, the requirement that $a^{2} V \ll 1$ means that this expansion is of little relevance beyond the leading term: terms of order $V$ in the numerator occur for arbitrarily large odd half-integral powers of $\mathcal{U}$ in the denominator, and similarly for terms of order $V^{2}$.

The question as to which linear combination of the modes (30) correspond to the respective solutions $\Psi_{1}=a^{(p-1) / 4} Y_{1}\left(\frac{1}{2} a^{2}\right)$ and $\Psi_{2}=a^{(p-1) / 4} Y_{2}\left(\frac{1}{2} a^{2}\right)$ of (8) can be resolved by expanding them in powers of $2 V z \equiv a^{2} V$. In particular, from (9)-(22) we find $Y_{1}(z)$ and $Y_{2}(z)$ are given by their definitions (19) and (20) where now 


$$
\begin{aligned}
y_{ \pm}(z) & =z^{ \pm \nu} \sum_{n=0}^{\infty}(-2 V z)^{n} \sum_{\ell=n}^{\infty} \boldsymbol{S}_{ \pm, \ell+[n / 2]}^{n} z^{2 \ell} \\
& =I_{ \pm \nu}(z)-2 V z \sum_{\ell=1}^{\infty} \sum_{j=0}^{\ell-1} \mathbf{C}_{ \pm, 2 \ell+1}^{1} \mathfrak{C}_{ \pm, 2 j}^{0} z^{2 \ell}+\ldots
\end{aligned}
$$

and

$$
\begin{aligned}
y_{*}(z)= & y_{+}(z) \ln (z) \\
& -2 z^{\nu} \sum_{n=0}^{\infty}(-2 V z)^{n} \sum_{\ell=1+[n / 2]}^{\infty} \sum_{\substack{i=1, \mathcal{D}_{2 \ell+n, i}=\ell}}^{h_{2 \ell+n}} \frac{z^{2 \ell}}{\prod_{j=1}^{\ell} \alpha_{j}^{i}\left(\alpha_{j}^{i}+2 \nu\right)}\left(\sum_{k=1}^{\ell} \frac{\left(\alpha_{k}^{i}+\nu\right)}{\alpha_{k}^{i}\left(\alpha_{k}^{i}+2 \nu\right)}\right) .
\end{aligned}
$$

We use the fact that

$$
I_{ \pm \nu}\left(\frac{1}{2} a^{2}\right)=\frac{1}{\sqrt{\pi} a} \exp \left(\frac{1}{2} a^{2}\right)\left[1+\mathrm{O}\left(a^{-2}\right)\right]
$$

and

$$
K_{\nu}\left(\frac{1}{2} a^{2}\right)=\frac{\sqrt{\pi}}{a} \exp \left(-\frac{1}{2} a^{2}\right)\left[1+\mathrm{O}\left(a^{-2}\right)\right]
$$

for large $a$ to compare the leading terms with (30), noting that the overall exponential $\exp \left[\frac{ \pm \mathcal{U}^{3 / 2}}{3 V}\right]$ in (30) may be expanded as $\exp \left[\frac{ \pm 1}{3 V} \mp \frac{1}{2} a^{2}\left(1+\mathrm{O}\left(a^{2} V\right)\right)\right]$. In this way we see, similarly to the observation in [11], that the leading order terms agree if we identify

$$
\Psi_{-} \propto \frac{1}{a^{(p-1) / 2}}\left[Y_{1}\left(\frac{1}{2} a^{2}\right)+\alpha Y_{2}\left(\frac{1}{2} a^{2}\right)\right]
$$

$\alpha \neq-1$, and

$$
\Psi_{+} \propto \frac{1}{a^{(p-1) / 2}}\left[Y_{2}\left(\frac{1}{2} a^{2}\right)-Y_{1}\left(\frac{1}{2} a^{2}\right)\right]
$$

An overall multiplicative factor $\exp \left(\frac{\mp 1}{3 V}\right)$ must be included in the constant of proportionality in (30) to cancel the overall $\phi$ dependence in the cases that the wave function can be normalized.

In the limit $x \rightarrow-\infty$, which corresponds to $a^{2} V \gg 1$, or $a \rightarrow \infty$, we may find asymptotic series which apply beyond leading order. In particular, the linearly independent modes are found to be

$$
\begin{aligned}
& \Psi_{-} \propto \frac{2}{a^{(p+1) / 2}(-\mathcal{U})^{1 / 4}}\left\{\cos \left[\frac{(-\mathcal{U})^{3 / 2}}{3 V}-\frac{\pi}{4}\right] A_{1}+\sin \left[\frac{(-\mathcal{U})^{3 / 2}}{3 V}-\frac{\pi}{4}\right] A_{2}\right\}, \\
& \Psi_{+} \propto \frac{1}{a^{(p+1) / 2}(-\mathcal{U})^{1 / 4}}\left\{-\sin \left[\frac{(-\mathcal{U})^{3 / 2}}{3 V}-\frac{\pi}{4}\right] A_{1}+\cos \left[\frac{(-\mathcal{U})^{3 / 2}}{3 V}-\frac{\pi}{4}\right] A_{2}\right\},
\end{aligned}
$$

where 


$$
\begin{aligned}
A_{1} \equiv 1 & -\left(\frac{77}{96}-\frac{1}{6} \mu\right)\left(\frac{5}{48}-\frac{1}{3} \mu\right) \frac{(2 V)^{2}}{(-\mathcal{U})^{3}}+\frac{1}{8}\left(\frac{16}{15} \mu-\frac{13}{3}\right) \mu \frac{(2 V)^{2}}{(-\mathcal{U})^{4}}+\frac{1}{10}\left(\frac{78}{35} \mu-\frac{445}{56}\right) \mu \frac{(2 V)^{2}}{(-\mathcal{U})^{5}} \\
& +\left(\frac{437}{192}-\frac{1}{12} \mu\right)\left(\frac{221}{144}-\frac{1}{9} \mu\right)\left(\frac{77}{96}-\frac{1}{6} \mu\right)\left(\frac{5}{48}-\frac{1}{3} \mu\right) \frac{(2 V)^{4}}{(-\mathcal{U})^{6}}+\frac{1}{12}\left(\frac{1208}{315} \mu-\frac{113}{9}\right) \mu \frac{(2 V)^{2}}{(-\mathcal{U})^{6}} \\
& +\ldots, \\
A_{2} \equiv \quad & \left(\frac{5}{48}-\frac{1}{3} \mu\right) \frac{2 V}{(-\mathcal{U})^{3 / 2}}+\frac{2}{5} \mu \frac{2 V}{(-\mathcal{U})^{5 / 2}}-\frac{3}{7} \mu \frac{2 V}{(-\mathcal{U})^{7 / 2}}+\frac{4}{9} \mu \frac{2 V}{(-\mathcal{U})^{9 / 2}} \\
& -\left(\frac{221}{144}-\frac{1}{9} \mu\right)\left(\frac{77}{96}-\frac{1}{6} \mu\right)\left(\frac{5}{48}-\frac{1}{3} \mu\right) \frac{(2 V)^{3}}{(-\mathcal{U})^{9 / 2}}-\frac{5}{11} \mu \frac{2 V}{(-\mathcal{U})^{11 / 2}} \\
& +\frac{1}{99}\left(\frac{11}{5} \mu^{2}-\frac{1471}{40} \mu+\frac{28231}{256}\right) \mu \frac{(2 V)^{3}}{(-\mathcal{U})^{11 / 2}}+\ldots,
\end{aligned}
$$

The phase in (37) and (38) is obtained by analytic continuation of the corresponding modes in (30), taking care with branch cuts similarly to the $\mu=0$ pure Airy function case [20]. To leading order, this of course agrees with the results obtained using the WKB connection formulae [11]. The no boundary wave function, $\Psi_{\mathrm{NB}}$, of Hartle and Hawking corresponds to $\Psi_{-}$, whereas Vilenkin's tunneling wave function, $\Psi_{\mathrm{TV}}$, corresponds to $\frac{1}{2} \Psi_{-}+i \Psi_{+}$, for the modes given by (37)-(40).

\section{DISCUSSION}

The physical interpretation of the series solutions to the de Sitter minisuperspace Wheeler-DeWitt equation (7), (8) yield no surprises in comparison with the conclusions which have already been reached in [11]. In particular, for operator orderings with $p \geq 1$ the contribution from $Y_{2}\left(\frac{1}{2} a^{2}\right)$ diverges at the origin, causing $\Psi$ to diverge also. (For $p<1$ the divergence is regulated by the prefactor.) Thus any boundary condition proposal which requires regularity of the wave function as $a \rightarrow 0$ must reduce to (35) with $\alpha=0$ if $p \geq 1$ in this particular model; i.e., it must coincide with the proposal of Hartle and Hawking to the level of the approximations which have been assumed. Since normalizability of the wave function at $a=0$ is an essential ingredient of calculations of the probability of inflation from quantum cosmology, the question of operator ordering cannot be ignored in such calculations. If the factor ordering $p=1$ is the natural one in the present model, as has been claimed [13, 14, then there are substantial problems for $\Psi_{\mathrm{TV}}$. While the Hartle-Hawking wave function, $\Psi_{\mathrm{NB}}$, has not been found to yield a definitive prediction of inflation for any potentials, $V(\phi)$, considered to date [21], the prognosis for $\Psi_{\mathrm{TV}}$ seems to be even more doubtful as an identification of type (1), (2) cannot be made in the first place if $p \geq 1$.

One might wonder whether these conclusions would be altered by the addition of noninflationary matter degrees of freedom to the model. Although strictly speaking the matter degrees of freedom themselves should be quantized, some indication of their bulk effect might be obtained by adding a perfect fluid source to the Wheeler-DeWitt equation [22 26]. For a 
homogeneous isotropic fluid of energy density, $\rho$, and pressure obeying an equation of state $P=w \rho$, we find that the minisuperspace Wheeler-DeWitt equation (8) is replaced by

$$
z^{2} \frac{\mathrm{d}^{2} y}{\mathrm{~d} z^{2}}+z \frac{\mathrm{d} y}{\mathrm{~d} z}+\left(2 V z^{3}+\beta z^{3(1-w) / 2}-k z^{2}-\nu^{2}\right) y=0,
$$

where the spatial curvature, $k$, is identified explicitly for the purpose of the discussion that follows, $z=\frac{1}{2} a^{2}$ and $\nu=\frac{1}{4}(p-1)$ as before. The normalization factor in (3) is now given in general by $\sigma^{2}=\kappa^{2} /(3 \mathcal{V}), \mathcal{V}$ being the 3-volume of the unit spatial hypersurface (with suitable topological identifications for $k=0,-1)$, and $\beta=2 \kappa^{2} \sigma^{2} a_{0}^{3(1+w)} /\left(3 \rho_{0}\right)$ for a matter source with energy density normalized to $\rho=\rho_{0}$ at $a=a_{0}$. For large values of $a$, the scalar potential term dominates irrespective of the other matter degrees of freedom or the spatial curvature, and the solutions approximate to a superposition of modes $a^{(1-p) / 2} J_{(p-1) / 6}\left(\frac{1}{3} a^{3} \sqrt{V}\right)$ and $a^{(1-p) / 2} Y_{(p-1) / 6}\left(\frac{1}{3} a^{3} \sqrt{V}\right)$ as was discussed in [1].

Now let us consider the properties of the solutions as $a \rightarrow 0$, which is important for normalization of the wave function. The case of stiff matter $(w=1)$ in general yields a singular wave function as $a \rightarrow 0$ for both modes, and hence any choice of boundary condition, the singularity being of infinitely oscillatory type if $\beta>\nu^{2}=\frac{1}{16}(p-1)^{2}$,

$$
\Psi \underset{a \rightarrow 0}{\sim} a^{(1-p) / 2}\left\{C_{1} \cos \left[\ln \left(\frac{1}{2} \sqrt{\beta-\nu^{2}} a^{2}\right)\right]+C_{2} \sin \left[\ln \left(\frac{1}{2} \sqrt{\beta-\nu^{2}} a^{2}\right)\right]\right\},
$$

where $C_{1,2}$ are constants. This is a pathological type of behaviour which does not distinguish between different boundary conditions for the wave function, and is presumably not of physical interest. Apart from this case, however, one may readily see that the conclusions of [11] are not altered in general by the presence of additional matter, since the indicial equation for series solutions about $a=0$, which primarily determines the properties of the solutions near $a=0$, is unaffected for $w<1$.

Consider the case of radiation $\left(w=\frac{1}{3}\right)$, for example, in which case (41) is still of Bôcher type $\{25\}$ [17]. As $a \rightarrow 0$ the leading order behaviour is given by that of the exact solutions to (41) with $V=0$, which are given by 25]

$$
\begin{aligned}
\Psi & =a^{-(2 \nu+1)}\left\{C_{1} M_{\beta / 2, \nu}\left(a^{2}\right)+C_{2} W_{\beta / 2, \nu}\left(a^{2}\right)\right\}, \\
& =\exp \left[-\frac{1}{2} a^{2}\right]\left\{C_{1} M\left(\nu+\frac{1}{2}-\frac{\beta}{2}, 1+2 \nu, a^{2}\right)+C_{2} U\left(\nu+\frac{1}{2}-\frac{\beta}{2}, 1+2 \nu, a^{2}\right)\right\},
\end{aligned}
$$

in terms of Whittaker functions and Kummer functions [27 respectively, for $k=+1$. Since $\exp \left[-\frac{1}{2} a^{2}\right] M\left(\nu+\frac{1}{2}, 1+2 \nu, a^{2}\right)=\Gamma[\nu+1]\left(\frac{2}{a}\right)^{2 \nu} I_{\nu}\left(\frac{1}{2} a^{2}\right)$ and $\exp \left[-\frac{1}{2} a^{2}\right] U\left(\nu+\frac{1}{2}, 1+2 \nu, a^{2}\right)=\pi^{-1 / 2} a^{-2 \nu} K_{\nu}\left(\frac{1}{2} a^{2}\right)$ [27], we of course retrieve the limiting $V=0$ solutions discussed in previous sections when $\beta=0$. The solutions for $k=-1$ and $V=0$ are likewise given by

$$
\begin{aligned}
\Psi= & \frac{1}{2} C_{1}\left\{\mathrm{e}^{i a^{2} / 2} M\left(\nu+\frac{1}{2}-i \frac{\beta}{2}, 1+2 \nu,-i a^{2}\right)+\mathrm{e}^{-i a^{2} / 2} M\left(\nu+\frac{1}{2}+i \frac{\beta}{2}, 1+2 \nu, i a^{2}\right)\right\} \\
+ & \frac{1}{2} C_{2}\left\{\mathrm{e}^{-i \pi \nu+i a^{2} / 2} U\left(\nu+\frac{1}{2}-i \frac{\beta}{2}, 1+2 \nu,-i a^{2}\right)+\mathrm{e}^{i \pi \nu-i a^{2} / 2} U\left(\nu+\frac{1}{2}+i \frac{\beta}{2}, 1+2 \nu, i a^{2}\right)\right\}
\end{aligned}
$$


which reduces to a superposition of ordinary Bessel function modes

$$
\Psi=a^{(1-p) / 2}\left\{B_{1} J_{\nu}\left(\frac{1}{2} a^{2}\right)+B_{2} Y_{\nu}\left(\frac{1}{2} a^{2}\right)\right\},
$$

when $\beta=0$, where relative to (44) $B_{1}=2^{2 \nu} \Gamma[\nu+1] C_{1}$ and $B_{2}=-\frac{1}{2} \sqrt{\pi} C_{2}$. In the spatially flat case, $k=0$, the solution to (41) with $V=0$ is given in terms of ordinary Bessel functions modes for all values of $\beta$ [24,26]

$$
\Psi=a^{(1-p) / 2}\left\{C_{1} J_{2 \nu}(\sqrt{\beta} a)+C_{2} Y_{2 \nu}(\sqrt{\beta} a)\right\} .
$$

In each of the cases (43), (44) and (46), the mode with coefficient $C_{1}$ is finite as $a \rightarrow 0$, whereas the mode with coefficient $C_{2}$ is proportional to $a^{-4 \nu}=a^{1-p}$ as $a \rightarrow 0$, which causes $\Psi$ to diverge for $p \geq 1$ unless $C_{2}=0$, in direct analogy to the radiation-free solutions $(\beta=0)$. Unfortunately, the requirement that Vilenkin's wave function, $\Psi_{\mathrm{TV}}$, is outgoing means that the coefficients of both modes must be non-zero, and $C_{2}=0$ is not possible for $\Psi_{\mathrm{TV}}$. In particular, in the model here Vilenkin's boundary condition requires that

$$
\operatorname{Re} \frac{i}{\Psi_{\mathrm{TV}}} \frac{\partial \Psi_{\mathrm{TV}}}{\partial a}>0
$$

in the Lorentzian region, which includes all values $a>0$ if $k=0,-1$. In the $k=0$ case (46), for example, the linear combination of modes which satisfies this condition is

$$
\Psi_{\mathrm{TV}} \propto a^{(1-p) / 2}\left\{J_{2 \nu}(\sqrt{\beta} a)-i Y_{2 \nu}(\sqrt{\beta} a)\right\}
$$

namely a Hankel function of the second kind, for which $\operatorname{Re} \frac{i}{\Psi_{\mathrm{TV}}} \frac{\partial \Psi_{\mathrm{TV}}}{\partial a}=2\left[\pi a\left(J_{2 \nu}{ }^{2}+Y_{2 \nu}{ }^{2}\right)\right]^{-1}$. Thus the problems faced by Vilenkin's wave function, especially with regard to the prediction of inflation [11], appear to be generic.

One must also conclude that great care must be exercised when making arguments based on the identification of probabilities according to (1), (2). In general, the contribution of the prefactor and choices which depend on Planck scale physics cannot be ignored when one is considering the problem of the nucleation of the universe.

Acknowledgement I would like to thank the Australian Research Council for financial support. 


\section{REFERENCES}

[1] S.W. Hawking and N.G. Turok, Phys. Lett. B425, 25 (1998); Phys. Lett. B432, 271 (1998).

[2] A.D. Linde, Phys. Rev. D58, 083514 (1998).

[3] S.W. Hawking and N.G. Turok, gr-qc/9802062.

[4] A. Vilenkin, Phys. Rev. D58, 067301 (1998).

[5] J.B. Hartle and S.W. Hawking, Phys. Rev. D28, 2960 (1983).

[6] A. Vilenkin, Phys. Rev. D33, 3560 (1986).

[7] A. Vilenkin, Phys. Rev. D37, 888 (1988).

[8] A.D. Linde, Zh. Eksp. Teor. Fiz. 87, 369 (1984) [Sov. Phys. JETP 60, 211 (1984)]; Lett. Nuovo Cimento 39, 401 (1984); Rep. Prog. Phys. 47, 925 (1984).

[9] J. Garriga and A. Vilenkin, Phys. Rev. D56, 2464 (1997).

[10] R. Bousso and S.W. Hawking, Phys. Rev. D54, 6312 (1996).

[11] N. Kontoleon and D.L. Wiltshire, Phys. Rev. D59, 063513 (1999).

[12] S.W. Hawking and D.N. Page, Nucl. Phys. B264, 185 (1986).

[13] J. Louko, Ann. Phys. (N.Y.) 181, 318 (1988); Class. Quantum Grav. 6, 1947 (1991).

[14] A.O. Barvinsky, Phys. Rep. 230, 237 (1993); Class. Quantum Grav. 10, 1985 (1993).

[15] A.O. Barvinsky and A.Yu. Kamenshchik, Class. Quantum Grav. 7, L181 (1990).

[16] A.O. Barvinsky, A.Yu. Kamenshchik Phys. Lett. B332, 270 (1994); A.O. Barvinsky, A.Yu. Kamenshchik and I.V. Mishakov, Nucl. Phys. B491, 387 (1997).

[17] P. Moon and D.E. Spencer, "Field theory handbook", (Springer, Berlin, 1961); J. Franklin Inst. 260, 41 (1955).

[18] T.M. Green, Math. Mag. 41, 13 (1968).

[19] I. Stewart, Sci. Am. 274, 92 (June 1996).

[20] R.B. Dingle, "Asymptotic expansions: Their derivation and interpretation" (Academic Press, London, 1973).

[21] A. Lukas, Phys. Lett. B347, 13 (1995).

[22] J.H. Kung, Gen. Relativ. Grav. 27, 35 (1995).

[23] M.L. Fil'chenkov, Phys. Lett. B354, 208 (1995).

[24] A. Carlini, D.H. Coule, and D.M. Solomons, Mod. Phys. Lett. A11, 1453 (1996); Int. J. Mod. Phys. A12, 3517 (1997).

[25] H. Rosu and J. Socorro, Nuovo Cimento B113, 119 (1998).

[26] D.H. Coule and J. Martin, Preprint gr-qc/9905056 (1999).

[27] M. Abramovitz and I.A. Stegun, Handbook of Mathematical Functions, (Dover, New York, 1965). 